\title{
Wachset und mehret Euch!
}

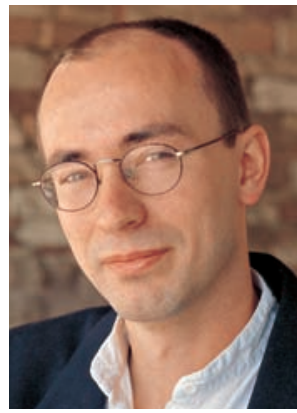

Dr. med. Dieter Schmid, Redaktionsleitung
Zählen Sie mal bis vier. Und das in einer Sekunde. Und dann nochmal und nochmal. Gar nicht so einfach, aber es geht. Wenn Sie gerade keine Uhr mit Sekundenanzeige haben, nehmen Sie einfach Ihren Puls. Der schlägt ziemlich genau ein Mal pro Sekunde. Haben Sie den Takt? Dann wissen Sie jetzt, wie viele Babys derzeit auf der Welt geboren werden: Genau in diesem Rhythmus ertönen auf dem Globus die empörten ersten Schreie von Neugeborenen. Pro Tag sind das etwa 350.000 „frischgebackene“ Erdenbürger. Genug zu tun also für die Ärzte, die werdende Mütter beim Gebärvorgang unterstützen möchten. Deutsche Medizinstudenten haben in dieser - global betrachtet - „florierenden Branche“ einen gewissen Standortnachteil. Mitunter fehlt es hierzulande nämlich am passenden „Anschauungsmaterial“. Zum Vergleich: Während auf den Philippinen zum Beispiel jede Frau drei bis vier Kinder auf die Welt bringt, sind das in Deutschland nur 1,3. Deshalb vermitteln wir Ihnen im Artikel „Kraftakt mit Happy End“ auf S. 36 so anschaulich wie irgend möglich das Grundlagenwissen, das Sie als potenzieller Geburtshelfer brauchen.

Was für Deutschland im Allgemeinen gilt, gilt für die deutsche Medizin im Besonderen: Es mangelt an Nachwuchs! Zahlreiche deutsche Fachverbände erwarten extreme personelle Engpässe. Beispiel: Der Hausärzteverband glaubt, dass bis 2020 etwa 15.000 Hausärzte fehlen werden. Eigentlich unverständlich, denn dieser Beruf ist facettenreich und familienfreundlich. Einen Eindruck von der breiten Palette hausärztlicher Tätigkeiten vermittelt die Broschüre „Perspektive Hausarzt“, die dieser Via medici beiliegt und die wir Ihnen wärmstens empfehlen möchten. Zudem lesen Sie in diesem Heft auf $\mathbf{S}$. 16 den PJ-Bericht „Husten, Schnupfen, Herzinfarkt“ von Tjark Schwemer. Er hat sein PJ-Wahltertial in der Allgemeinmedizin absolviert - und war danach hellauf begeistert.

Am Fach Medizin an sich liegt es also sicher nicht, dass in Deutschland Ärzte fehlen. Einer der Hauptgründe ist, dass viele junge Mediziner der Perspektive einer Anstellung in einer hiesigen Klinik so wenig abgewinnen können, dass sie abwandern entweder in nichtkurative Bereiche oder ins Ausland. Laut unserer diesjährigen PJ-Umfrage (S. 20) planen $20 \%$ der jungen Mediziner, zumindest einen Teil ihrer Ausbildung im Ausland zu verbringen. $28 \%$ spielen mit diesem Gedanken. Aufschlussreicher Kommentar eines Teilnehmers: „Ach, Leute. Macht mal was! Wir sind sonst ALLE weg. Ich habe mittlerweise Angst, zum Arzt zu gehen. Wenn die alle genauso wenig gelernt haben. Oh, mein Gott! “ Mein Tipp: Lassen Sie sich nicht entmutigen! In den vier Minuten, in denen Sie dieses Editorial gelesen haben, sind auf der Erde fast tausend Menschen geboren worden. Alles potenzielle Patienten, die vielleicht irgendwann Ihre Hilfe benötigen ...

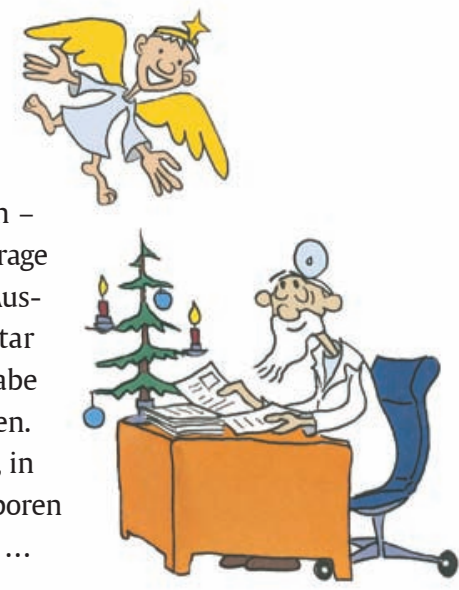

Eine frohe Weihnachtszeit wünscht Ihnen

Ihr

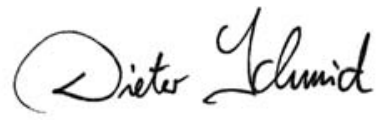

„Fürchte Dich nicht! Siehe, ich verkündige Dir große Freude, denn heute wurde Dir Dein NACHFOLGER geboren ....

\section{ONLINE-UMFRAGE}

\section{Lernen vor Prüfungen:} Wie viele Stunden sind normal?

Wenn Examina anstehen, verwandeln sich die meisten Medizinstudenten in eine Art Leistungssportler in Sachen Lernen. Aus gutem Grund denn binnen weniger Wochen oder Monate muss eine riesige Stoffmenge bewältigt werden. Dabei meistert jeder diese Aufgabe ein bisschen anders: Die einen setzen auf Zeitmanagement und ausgefeilte Lernpläne (S. 12), andere leben und lernen in den Tag hinein. Die einen vergraben sich vom frühen Morgen bis späten Abend zum Pauken am Schreibtisch, andere sind überzeugt, dass konzentriertes, kurzes Lernen effektiver ist, weil „irgendwann sowieso nichts mehr in den Kopf passt“. Welcher Philosophie hängen Sie an?

- Wie viele Stunden pro Tag lernen Sie in der heißen Phase vor wichtigen Prüfungen?

Verraten Sie uns, wie Ihr Lernplan aussieht, unter: www.thieme.de/viamedici/aktuelles/aktion/ miniumfrage5_09.html
- Ergebnis der Umfrage in Via medici 4.09: Wir fragten, ob Sie die Einführung eines „Bachelors der Medizin“ befürworten. Knapp 90\% der Teilnehmer lehnen die Reform ab, nur $10 \%$ fänden sie gut. Ein Befürworter stellt die Frage: „Warum sollte es nicht möglich sein nach acht Semestern in die Forschung zu gehen?“ Ein Kritiker kontert: „Die Fülle an medizinischem Wissen lässt sich in diesem kurzen Studium nicht vermitteln, ohne an Inhalten zu sparen. Der Bachelor würde zudem die Zahl approbierter Ärzte dezimieren. “ Alle Kommentare unter: .../aktuelles/aktion/miniumfrage4_09.html 